\title{
"Particle-free" Magnetic Actuation of Droplets on Superhydrophobic Surfaces Using Dissolved Paramagnetic Salts
}

\author{
Lili Mats, Fiona Logue and Richard D. Oleschuk ${ }^{*}$ \\ Department of Chemistry, Queen's University, \\ 90 Bader Lane, Kingston, Ontario, Canada, K7L 3N6
}

- Scanning electron microscope image of the commercial fluorinated silica nanoparticle surface

- Determination of water contact angle using ImageJ software

- Roll-off angles and droplet base for10 $\mu \mathrm{L}$ droplets containing different concentrations of paramagnetic salts

Corresponding author email: oleschuk@queensu.ca 


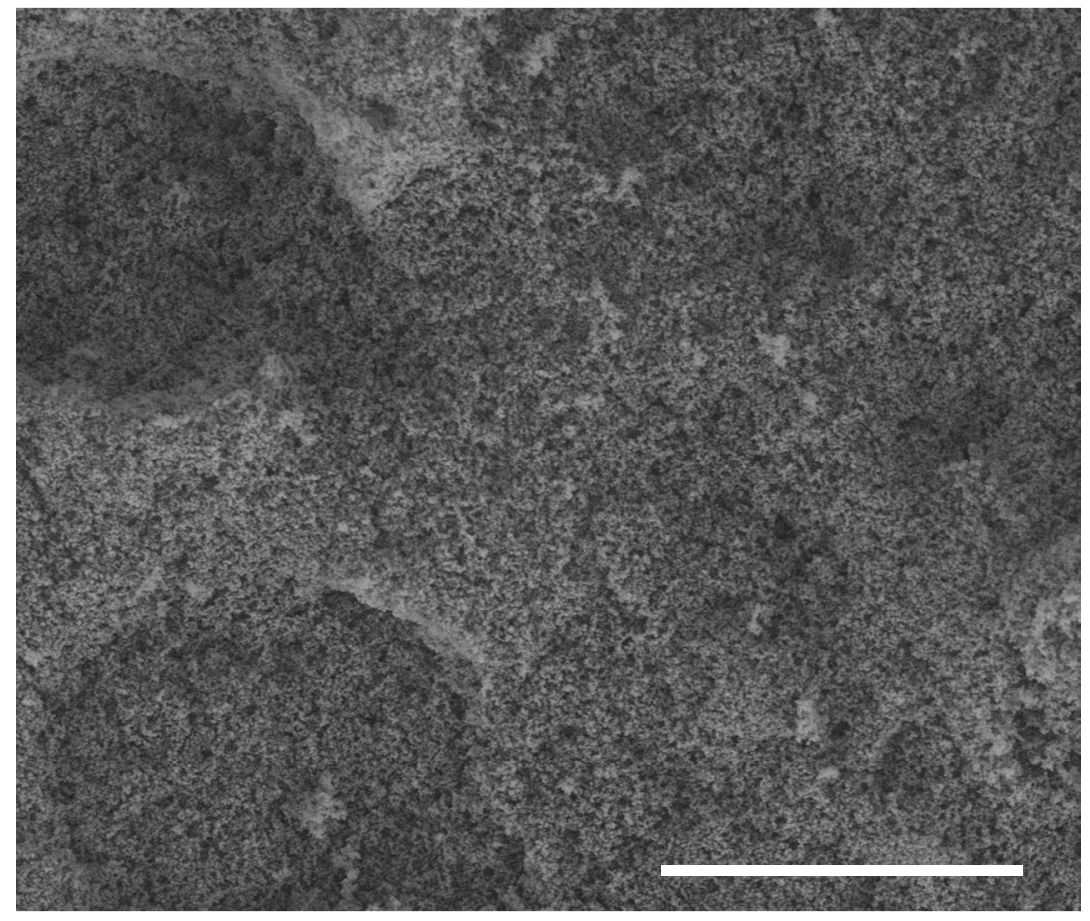

Figure S1: SEM photograph of UED surface on a glass slide, demonstrating roughness on micro- and nanoscale due to presence of fluorinated silica nanoparticles (scale bar $=10 \mu \mathrm{m}$ ).

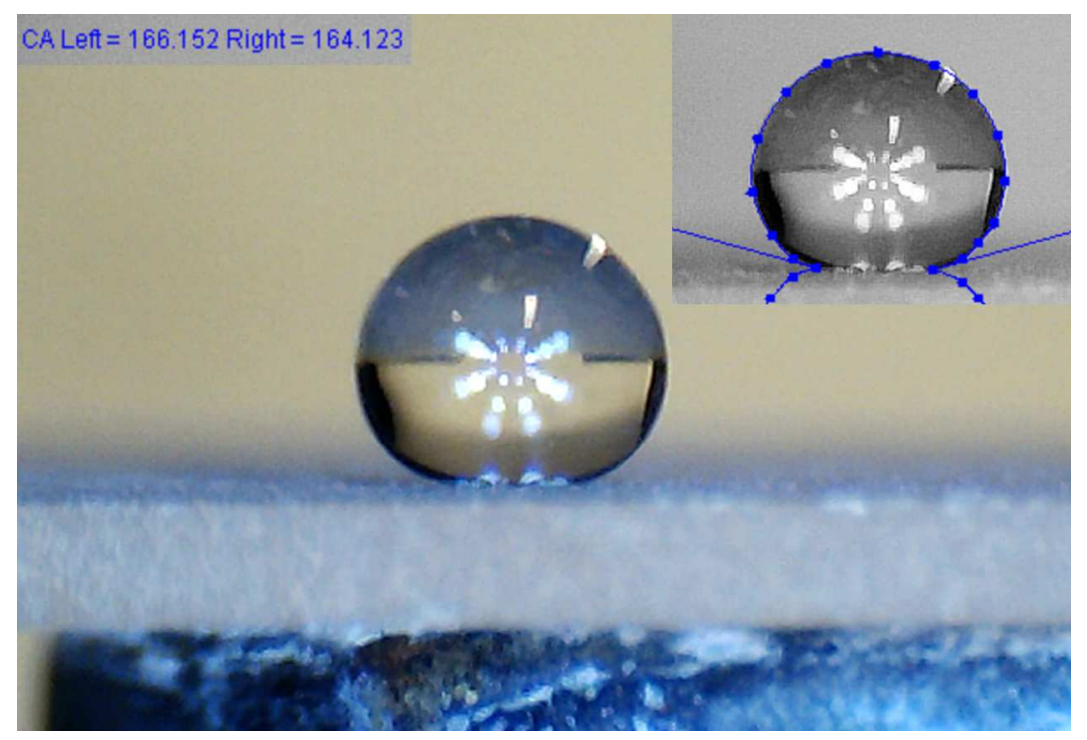

Figure S2: A $10 \mu \mathrm{L}$ droplet of $0.3 \mathrm{M} \mathrm{MnCl}_{2} \cdot 4 \mathrm{H}_{2} \mathrm{O}$ salt over superhydrophobic UED surface held by a magnet, where contact angle is $\sim 165^{\circ}$. The insert shows the measurement from the imageJ software. 


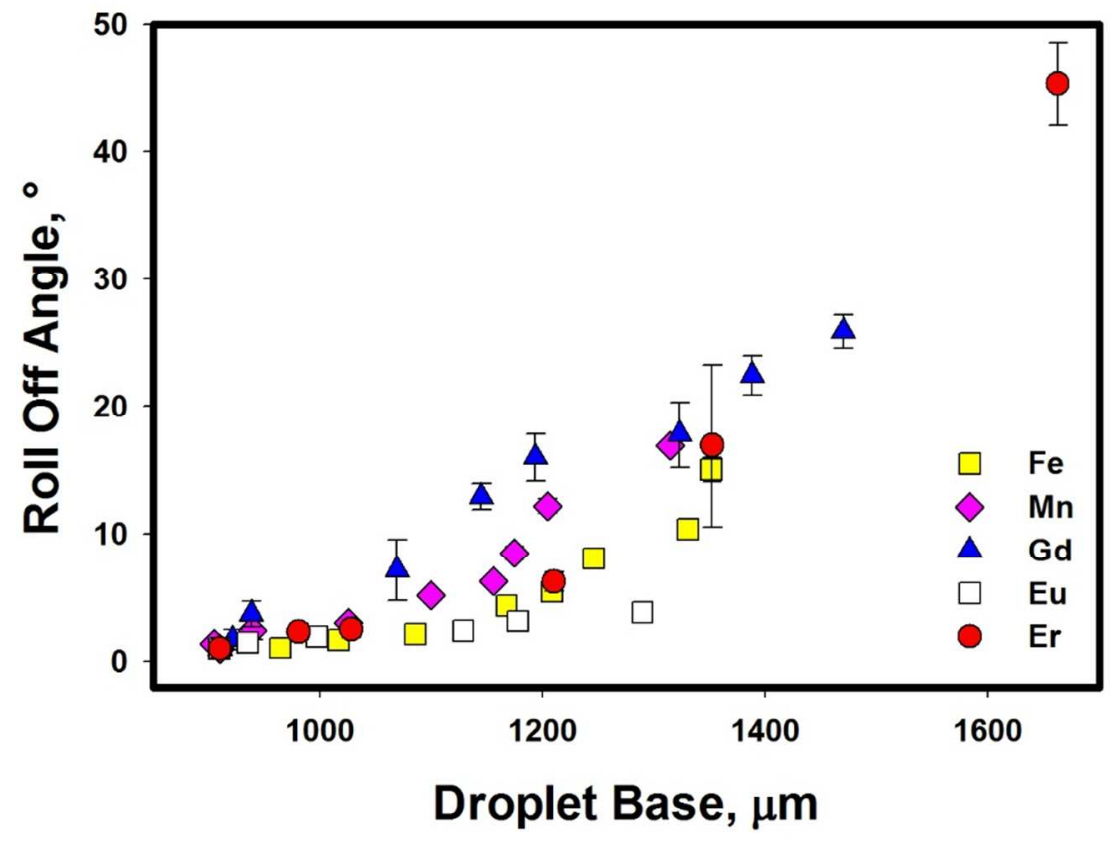

Figure S3: Roll-off angles and droplet base for $10 \mu \mathrm{L}$ droplets containing different concentrations of paramagnetic salts on a commercial superhydrophobic coating measured using $2.1 \mathrm{kG}$ disk magnet. 